\title{
Asymptotic behaviour of the porous media equation in domains with holes
}

\author{
CRISTINA BRÄNDLE` FERNANDO QUIRÓS ${ }^{\ddagger}$ AND JUAN LUIS VÁZQUEZ ${ }^{\S}$ \\ Departamento de Matemáticas, Universidad Autónoma de Madrid, 28049 Madrid, Spain
}

[Received 27 January 2006 and in revised form 21 December 2006]

\begin{abstract}
The paper deals with the asymptotic behaviour of solutions to the porous media equation, $u_{t}=\Delta u^{m}$, $m>1$, in an exterior domain, $\Omega$, which excludes one or several holes, and with zero Dirichlet data on $\partial \Omega$. When the space dimension is three or more this behaviour is given by a Barenblatt function away from the fixed boundary $\partial \Omega$ and near the free boundary. The asymptotic behaviour of the free boundary is given by the same Barenblatt function. On the other hand, if the solution is scaled according to its decay factor, away from the free boundary and close to the holes it behaves like a function whose $m$-th power is harmonic and vanishes on $\partial \Omega$. The height of such a function is determined by matching with the Barenblatt solution representing the outer behaviour. The inner and the outer behaviour can be presented in a unified way through a suitable global approximation.
\end{abstract}

2000 Mathematics Subject Classification: 35B40, 35R35, 35K65.

Keywords: Porous media equation; exterior domain; asymptotic behaviour; free boundary; matched asymptotics.

\section{Introduction}

Let $G \subset \mathbb{R}^{N}$ be a bounded open set with smooth boundary and let $\Omega=\mathbb{R}^{N} \backslash \bar{G}$. We do not assume $G$ to be connected, so that it may represent one or several holes in an otherwise homogeneous medium. Our goal is to study the large-time behaviour of the solution to the porous media equation (PME for short) in that exterior domain with zero data on the boundary,

$$
\begin{cases}u_{t}=\Delta u^{m}, & (x, t) \in \Omega \times(0, \infty), \\ u(x, t)=0, & (x, t) \in \partial \Omega \times(0, \infty), \\ u(x, 0)=u_{0}(x), & x \in \Omega\end{cases}
$$

where $m>1$. In order to simplify the presentation we assume that $\Omega$ is connected, since the general case follows from that case and known results [22]. By smooth boundary we mean that it is a $C^{2, \alpha}$ embedded manifold, which is a standard assumption in the literature, though the results hold under less regularity at the cost of longer proofs.

As for the initial data, we assume that $u_{0}$ is in $L^{1}(\Omega)$, nonnegative in $\Omega$, not identically zero and compactly supported in $\bar{\Omega}$. The last assumption implies that the support of $u(\cdot, t)$ remains bounded for any later time, $t>0$ (finite propagation property, which follows easily by comparison with the solution of the problem in the whole space). This allows us to study the behaviour in time of the free boundary, $\Gamma(t)=\partial\{x \in \Omega: u(x, t)>0\} \backslash \partial \Omega$, which is an important topic in porous media flows.

\footnotetext{
†E-mail: cristina.brandle@uam.es

‡E-mail: fernando.quiros@uam.es

${ }^{\S}$ E-mail: juanluis.vazquez@uam.es
} 
Due to the $L^{1}-L^{\infty}$ regularizing effect, [6], [23], [25], we may assume without loss of generality that $u_{0} \in L^{\infty}(\Omega)$, and then solutions are continuous for all $t>0$. A general reference for the theory of the PME is the monograph [24].

The asymptotic behaviour of the solution to the present problem (1.1) was studied by King in [16], both for $N=3$ and $N=2$. However, his calculations are formal and restricted to radially symmetric solutions. The aim of this paper is to perform a complete analysis of the issue when $N \geqslant 3$ for general domains and data. The restriction on the dimension will be assumed hereafter, since the situation for $N=1,2$ is different. As a first step, in Section 2 we construct sub- and supersolutions that will allow us to identify the decay and expansion rates of the solution. We show that $u$ decays as $O\left(t^{-\alpha}\right)$ while its support expands like $O\left(t^{\beta}\right)$, where

$$
\alpha=\frac{N}{N(m-1)+2}, \quad \beta=\frac{1}{N(m-1)+2}
$$

turn out to be the self-similarity exponents corresponding to the source-type solutions of the PME, also known as Barenblatt solutions. We scale the solution according to these rates,

$$
v_{\text {out }}(y, t)=t^{\alpha} u\left(y t^{\beta}, t\right),
$$

and prove that $v_{\text {out }}$ converges as $t \rightarrow \infty$ to the profile $F_{C_{\star}}$ of a particular Barenblatt solution, $B_{C_{\star}}(x, t)=t^{-\alpha} F_{C_{\star}}\left(|x| t^{-\beta}\right)$. The precise value of $C_{\star}$ is determined from the initial data thanks to an explicit conservation law. Convergence is uniform in sets $|y| \geqslant \delta$, i.e., in a wide exterior region up to the free boundary, which is called in Matched Asymptotics the outer limit (see Theorem 4.1).

The asymptotic mass of $u$ coincides with the mass of the Barenblatt function $B_{C_{\star}}$ (see Corollary 4.2). The amount of mass that is lost through the boundary is given by the projection of the initial data on a function $\Phi$ which is the normalized harmonic function that measures the capacity of $G$ (see formula (4.6). This is the only influence of the hole structure on the outer asymptotic behaviour in first approximation.

As a consequence of the convergence of both $v_{\text {out }}$ and the mass, we prove that the free boundary of $u$ behaves for large times as the free boundary of the Barenblatt function $B_{C_{\star}}$ (the one that gives the outer behaviour; see Corollary 4.3 .

In order to complete the study we must also consider what happens in the region near the holes (the so-called inner limit). The scaling in this case is simpler: we only have to amplify the solution, keeping the space variable fixed (i.e., a quasi-stationary situation). We prove that $v_{\text {in }}(x, t)=t^{\alpha} u(x, t)$ converges to a stationary state, $H_{C}(x)=C^{m /(m-1)} H(x)$, where $H=1-\Phi$ is the unique continuous solution of

$$
\Delta H=0, x \in \Omega, \quad H=0, x \in \partial \Omega, \quad H \rightarrow 1 \text { uniformly as }|x| \rightarrow \infty .
$$

The existence of $H$ is proved by considering solutions in the intersection of $\Omega$ with balls of increasing radius, which take boundary data equal to 1 in the boundary of the ball. The sequence of solutions is monotone and bounded, hence the limit is harmonic, [10]. Uniqueness is an immediate consequence of the maximum principle. The free constant $C$ is adjusted through matching with the Barenblatt function which gives the outer behaviour. It turns out that $C=C_{\star}$. See Section 5 for complete details.

A combination of the inner and outer descriptions allows us to write a global uniform approximation for the large-time behaviour of the solution (cf. Theorem 6.1). In the overlapping region

$$
1 / \delta<|x|<\delta t^{\beta}, \quad \delta \text { small, }
$$


both the inner and the outer limit differ very little from the global approximation, and hence between themselves. Therefore, a posteriori one realizes that the outer limit holds uniformly for $|x|>1 / \delta$ and the inner one for $|x| \leqslant \delta t^{\beta}$.

In order to illustrate the theory, in Section 7 we show some numerical computations for a radial example. We present some conclusions and comments in the last section, Section 8

Let us review some precedents: in [18], two of the authors study the same exterior problem, but with nontrivial boundary data, $g$. Assuming that $g$ is time independent, the inner limit of the solution stabilizes to $H^{1 / m}$, where $H$ is a harmonic function in the exterior domain with boundary data $g$ and decaying at infinity. The outer behaviour is given by a self-similar solution of the PME which is singular at $x=0$. In contrast to the case of zero boundary data, in this case the inner limit is completely determined by $g$ and the matching is needed in order to properly describe the outer limit. In other words, the sense of the implications in the matching process is reversed.

Let us also recall that the one-dimensional problem has been studied by Kamin and one of the authors in [15]. After an odd extension, the problem is identified as the PME equation with changing-sign initial data with zero mass, $\int_{\mathbb{R}} u_{0}=0$. The solution is shown to converge uniformly to a self-similar antisymmetric profile, a so-called dipole solution, introduced by Barenblatt and Zel'dovich in [5]. More precisely,

$$
\lim _{t \rightarrow \infty} t^{\alpha}|u(x, t)-D(x, t)|=0,
$$

uniformly in $\mathbb{R}$, where

$$
D(x, t)=t^{-\alpha} F\left(x t^{-\beta}\right), \quad \alpha=\frac{1}{m}, \quad \beta=\frac{1}{2 m},
$$

for a certain odd function $F$.

Notice that for $N=1$ there is no need to consider the outer and the inner region separately, since the dipole is already a global approximation. Besides, these scaling exponents do not match the exponents in (1.2), thus showing the different effect of the hole in one and more than two dimensions. The main physical difference is reflected in the fact that for $N \geqslant 3$ the asymptotic mass is not zero, while for $N=1$ it goes to zero like a power of time, $\int_{\mathbb{R}} u(x, t) \mathrm{d} x=O\left(t^{-1 / 2 m}\right)$. Dimension $N=2$ studied in [11] exhibits a transition behaviour where the mass goes to zero at a logarithmic rate.

Throughout the paper $C, c, c_{1}, \ldots$ denote positive constants that may change from one line to another when no confusion is to be feared. We denote by $\mathcal{B}_{r}\left(x_{0}\right)$ the open ball with radius $r$ and centre at $x_{0}$. We use $\operatorname{supp}(f)$ to refer to the spatial support of a function $f$.

NotE. After completion of this work, and during a conference held in Będlewo (Poland), we were informed of the paper by Profs. Gilding and Goncerzewicz on the same subject, [11]. Let us briefly comment on the differences. They obtain the outer behaviour both for $N \geqslant 3$ and for $1<N<3$, and they also treat fractional dimensions in the radial cases. On the other hand, the present manuscript contains several new topics, namely, the description of the inner behaviour (see Section 57, the rate of decay of the mass to its asymptotic limit, which is expressed in terms of the capacity of the hole, and the global approximation. The proof of the common result is quite different: In [11] the authors first prove the result for radial problems, including fractional spatial dimensions. The radial result is later applied to prove the general case. In this paper the general case is proved directly using a scaling argument. In [11] the holes are simply connected, an assumption that we do not need. 


\section{Preliminaries}

\subsection{Definitions}

We can find in the literature several concepts of solution for the PME (cf. [24]). Hence, we make precise the one we are dealing with. Let $Q_{T}=\Omega \times(0, T]$.

Definition 1 A function $u \in C\left((0, T] ; L^{1}(\Omega)\right) \cap L^{\infty}\left(Q_{T}\right)$ defined in $\bar{Q}_{T}$ is a weak solution of Problem 1.1 on $[0, T]$ if for any test function $\phi \in C^{2,1}\left(\bar{Q}_{T}\right)$, compactly supported in $\bar{Q}_{T}$, with $\phi=0$ on $\partial \Omega \times(0, T], u$ satisfies the integral identity

$$
\int_{\Omega} u(x, t) \phi(x, t) \mathrm{d} x=\int_{0}^{t} \int_{\Omega}\left\{u^{m}(x, t) \Delta \phi(x, t)+u(x, t) \phi_{t}(x, t)\right\} \mathrm{d} x \mathrm{~d} t+\int_{\Omega} u_{0}(x) \phi(x, 0) \mathrm{d} x
$$

for any $0 \leqslant t \leqslant T$. We say that $u$ is a weak solution of $(1.1)$ on $[0, \infty)$ if it is a solution, in the previous sense, on any $[0, T]$.

We define weak sub- and supersolutions as usual, i.e., by replacing in the definition of solution the $=$ sign by $a \leqslant$ or $\geqslant$, respectively, and considering only nonnegative test functions $\phi \geqslant 0$. See [24. Chapters 5, 6 and 9].

The existence and uniqueness of weak solutions is well-known in the case of bounded domains, and is extended to the case of unbounded domains in [24]. More precisely, for general data $u_{0} \in$ $L^{1}(\Omega)$ it can be shown, solving Dirichlet problems in bounded domains $\Omega_{n}=\Omega \cap \mathcal{B}_{n}(0)$ and considering suitable approximations $u_{0 n}$ of $u_{0}$, defined in $\Omega_{n}$, that solutions to (1.1) exist. Even more, note that we are considering solutions with compactly supported initial data, which leads to compact support in space for all times. Hence, the standard construction on bounded domains is enough to show that the solution exists on $[0, T]$ for all finite $T$.

Uniqueness and comparison follow easily from the next proposition.

Proposition 2.1 Let $u$ be a weak subsolution of Problem (1.1) with initial data $u_{0}$ and $\hat{u}$ a weak supersolution with initial data $\hat{u}_{0}$. Then for each $0 \leqslant t \leqslant T$,

$$
\int_{\Omega}[u(x, t)-\hat{u}(x, t)]_{+} \mathrm{d} x \leqslant \int_{\Omega}\left[u_{0}(x)-\hat{u}_{0}(x)\right]_{+} \mathrm{d} x,
$$

where $[r]_{+}=\max \{r, 0\}$.

The proof is analogous to the one already given in [3] for one-dimensional bounded domains.

Moreover, the standard theory of weak solutions provides us with estimates of the form

$$
\int_{0}^{T} \int_{\Omega}\left|\nabla u^{m}\right|^{2} \mathrm{~d} x d t \leqslant C \int_{\Omega} u_{0}^{m+1}(x) \mathrm{d} x
$$

For these properties cf. [24, Chapters 5 and 9].

We will also need the concept of local weak solution, where the initial and boundary conditions are not considered.

Definition 2 We say that $u \in C\left((0, T] ; L^{1}(\Omega)\right) \cap L^{\infty}\left(Q_{T}\right)$ for some $T>0$ is a local weak solution if $u$ satisfies the integral identity

$$
\int_{0}^{T} \int_{\Omega}\left\{u^{m}(x, t) \Delta \phi(x, t)+u(x, t) \phi_{t}(x, t)\right\} \mathrm{d} x \mathrm{~d} t=0
$$

for any test function $\phi \in C^{2,1}\left(Q_{T}\right)$ compactly supported in $Q_{T}$. 


\subsection{Conservation law}

The role of the standard mass conservation is played here by a modification in the form of a weighted mass conservation. Let $f$ be a harmonic function in $\Omega$ such that $f=0$ on $\partial \Omega$. As in [16], we introduce

$$
I(t)=\int_{\Omega} f(x) u(x, t) \mathrm{d} x .
$$

Integrating by parts in $\Omega$, since $u$ has compact support, we get formally

$$
\frac{\mathrm{d} I}{\mathrm{~d} t}=\int_{\Omega} f \Delta u^{m}=\int_{\partial \Omega}\left(f \frac{\partial u^{m}}{\partial v}-u^{m} \frac{\partial f}{\partial v}\right),
$$

where $v$ is the outward normal. Since $u^{m}$ and $f$ are zero on $\partial \Omega$, we conclude that $I$ is an integral invariant,

$$
\frac{\mathrm{d} I}{\mathrm{~d} t}=0 .
$$

The rigorous justification can be given by one of the two standard tricks used in the porous medium theory: raising the data by $\varepsilon$ so that the equation is no more degenerate for these data, or regularizing the nonlinearity $\Phi(u)=u^{m}$ into some smooth and nondegenerate $\Phi_{\varepsilon}$. We then pass to the limit $\varepsilon \rightarrow 0$. Such techniques are described in detail in [24].

\section{Sub- and supersolutions}

I. Supersolutions. As supersolutions we will use the Barenblatt functions

$$
B_{C}(x, t)=t^{-\alpha}\left(C-\frac{\beta(m-1)}{2 m} \frac{|x|^{2}}{t^{2 \beta}}\right)_{+}^{1 /(m-1)}=t^{-\alpha} F_{C}(|\xi|),
$$

with $C>0$ and $\xi=x t^{-\beta}$, which are source-type solutions of the PME in the whole space. As is well-known (cf. [2, 21, 24]), these are weak solutions of the equation for $t \geqslant \tau>0$, which have as initial trace a multiple of the Dirac delta, $M \delta(x)$. The parameter $C$ is a function of the total mass of $B_{C}$, more precisely, the mass $M_{C}=\int_{\mathbb{R}^{N}} B_{C}(x, t) \mathrm{d} x$ is related to $C$ by

$$
M_{C}=k(m, N) C^{1 / 2(m-1) \beta},
$$

where

$$
k(m, N)=2 \pi^{N / 2} \beta\left(\frac{2 m}{\beta(m-1)}\right)^{N / 2} \frac{\Gamma(\sigma)}{\Gamma(\sigma /(2 \beta))}, \quad \sigma=\frac{1}{m-1} .
$$

Given any positive time $\bar{t}$, we choose $C$ large so that $B_{C}(\cdot, \bar{t})$ lies above $u(\cdot, \bar{t})$. Recall that $u(\cdot, \bar{t})$ is bounded and compactly supported.

II. Subsolutions. The Barenblatt functions cannot be used as subsolutions of the problem: though a suitable translation allows putting them below $u$ at some time for $C$ small, they will eventually become positive at the fixed boundary $\partial \Omega$. To avoid this difficulty with the boundary condition, we may consider

$$
H_{A}(x, t)=A t^{-\alpha}\left(1-\left(\frac{R_{-}}{|x|}\right)^{N-2}\right)_{+}^{1 / m} .
$$


It has the expected decay factor and its $m$-th power is harmonic in its support. Hence it is a subsolution of the PME. However, it is impossible to put it below $u$ at any time, because its does not have compact support. The idea is then to take a combination of both subsolutions. But they intersect with the "wrong angle". Therefore, we have to modify $H_{A}$ slightly before "gluing" them. We take a delay $\tau>0$ and consider

$$
\begin{aligned}
\widehat{H}_{A(t), \tau}(x, t) & =A(t)(t+\tau)^{-\alpha}\left(1-\left(\frac{R_{-}}{|x|}\right)^{N-2}-\frac{a\left(|x|-r_{1}\right)_{+}^{4}}{(t+\tau)^{4 \gamma}}\right)_{+}^{1 / m}, \\
B_{C_{0}, \tau}(x, t) & =(t+\tau)^{-\alpha}\left(C_{0}-\frac{\beta(m-1)}{2 m} \frac{|x|^{2}}{(t+\tau)^{2 \beta}}\right)_{+}^{1 /(m-1)},
\end{aligned}
$$

where $R_{-}, r_{1}, a, C_{0}$, and $\gamma$ are positive constants, with $\gamma<\beta$. For $\sigma>0$, we set $A(t)=$ $2 C_{0}^{1 /(m-1)}\left(1+(t+\tau)^{-\sigma}\right)$, so that the maximum of $\widehat{H}_{A(t), \tau}$ is always greater than the maximum of $B_{C_{0}, \tau}$. Let $R_{+}(t)$ be the radius of the outer interface of $\widehat{H}_{A(t), \tau}$ and $B_{+}(t)$ the radius of the interface of $B_{C_{0}, \tau}$. We will see that if the parameters are selected appropriately, then $\widehat{H}_{A(t), \tau}$ and $B_{C_{0}, \tau}$ intersect at a distance $r_{\star}(t)$ that satisfies $r_{\star}(t)<R_{+}(t)$, with the correct angle, as in Figure 1 .

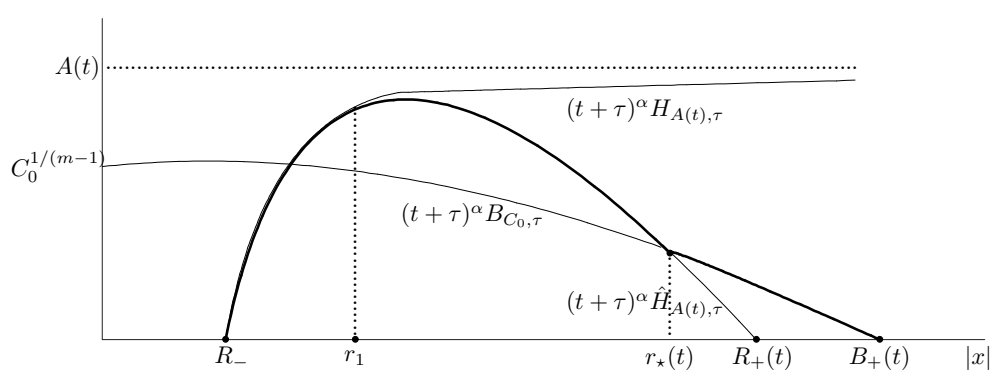

FIG. 1. Subsolution $\varphi_{C_{0}, \tau}$.

Hence, we define

$$
\varphi_{C_{0}, \tau}(x, t)= \begin{cases}0, & |x|<R_{-} \text {or }|x|>B_{+}(t), \\ \widehat{H}_{A(t), \tau}(x, t), & R_{-} \leqslant|x| \leqslant r_{\star}(t), \\ B_{C_{0}, \tau}(x, t), & r_{\star}(t) \leqslant|x| \leqslant B_{+}(t),\end{cases}
$$

which turns out to be the needed subsolution.

Lemma 3.1 There exist a time $t_{0}>0$ large, $C_{0}$ small, $\sigma<4 \gamma-1$ and a delay $\tau$ such that $\varphi_{C_{0}, \tau}(x, t)<u(x, t)$ for $t \geqslant t_{0}$.

Proof. We perform the proof in three steps. We first show that there is a time $\bar{t}$ such that $\varphi_{C_{0}, \tau}$ is a subsolution to the PME for $t \geqslant \bar{t}$. Then we show that there exist a time $t_{0} \geqslant \bar{t}$, a delay $\tau$ and constants $R_{-}, r_{1}, a$ and $C_{0}$ such that $u\left(x, t_{0}\right)$ is above $\varphi_{C_{0}, \tau}\left(x, t_{0}\right)$. The result then follows by comparison, by showing that $\varphi_{C_{0}, \tau}(x, t)$ is well defined for $t \geqslant t_{0}$.

Step 1. Since $B_{C_{0}, \tau}$ and $H_{A(t), \tau}$ are subsolutions of the PME, by definition of $\widehat{H}_{A(t), \tau}$ we only have to prove that it is a subsolution if $r_{1}<|x|<r_{\star}$ and $t \geqslant \bar{t}$; i.e., defining $r=|x|$ and 
$\widehat{H}(r, t)=\widehat{H}_{A(t), \tau}(x, t)$ we have to show that

$$
\widehat{H}_{t}-\frac{N-1}{r}\left(\widehat{H}^{m}\right)_{r}-\left(\widehat{H}^{m}\right)_{r r} \leqslant 0, \quad r_{1}<r<r_{\star}, t \geqslant \bar{t} .
$$

Since $\sigma<4 \gamma-1$, this inequality is satisfied if $\bar{t}$ is large.

Step 2. Let $\mathcal{A}=\mathcal{B}_{A_{+}}(0) \backslash \mathcal{B}_{A_{-}}(0)$ be an annulus such that for a fixed time $t_{0} \geqslant \bar{t}$, large enough, $\overline{\mathcal{A}} \subset \operatorname{Int}\left(\operatorname{supp}\left(u\left(\cdot, t_{0}\right)\right)\right.$ ) (see [4]). We choose $R_{-}=A_{-}$and $A_{-}<r_{1}<A_{+}$and then select $C_{0}$ which measures the height of $\varphi_{C_{0}, \tau}$ in order to have $u_{0}$ above $\varphi_{C_{0}, \tau}$ at $t_{0}$; i.e., we want $A(t)<\min u\left(\cdot, t_{0}\right)$ inside $\mathcal{A}$. We still have two free parameters, namely $a$ and $\tau$. Since we want $\operatorname{supp}\left(\varphi_{C_{0}, \tau}\left(\cdot, t_{0}\right)\right)=\left[R_{-}, B_{+}\left(t_{0}\right)\right] \subset \operatorname{Int}\left(\operatorname{supp}\left(u\left(\cdot, t_{0}\right)\right)\right)$, we impose first that

$$
B_{+}\left(t_{0}\right)=\left(c C_{0}\right)^{1 / 2}\left(t_{0}+\tau\right)=A_{+} .
$$

This determines the value of $\tau$. The next step consists in determining $a$. To this end we use the fact that $R_{+}(t)$ satisfies

$$
R_{+}(t)<r_{1}+\frac{(t+\tau)^{\gamma}}{a^{1 / 4}} .
$$

Hence, since we need $R_{+}\left(t_{0}\right)<B_{+}\left(t_{0}\right)$, it is enough to choose the free parameter $a$ such that $r_{1}+\left(t_{0}+\tau\right)^{\gamma} / a^{1 / 4}<B_{+}\left(t_{0}\right)$.

Step 3. The construction of $\varphi_{C_{0}, \tau}$ requires that $R_{+}(t)<B_{+}(t)$ in order to have the right intersection angle at $r_{\star}$. Let us see then that $r_{1}+(t+\tau)^{\gamma} / a^{1 / 4}<B_{+}(t)$. Define

$$
g(t)=r_{1}+\frac{(t+\tau)^{\gamma}}{a^{1 / 4}}-B_{+}(t) .
$$

From the previous step we know that $g\left(t_{0}\right)<0$. Assume that there is a first time $t_{1}$ where $g\left(t_{1}\right)=0$. Then, since $\gamma<\beta$,

$$
g^{\prime}\left(t_{1}\right)=\frac{\gamma\left(t_{1}+\tau\right)^{\gamma-1}}{a^{1 / 4}}-B_{+}\left(t_{1}\right) \beta\left(t_{1}+\tau\right)^{-1}=B_{+}\left(t_{1}\right)\left(t_{1}+\tau\right)^{-1}\left(\gamma-\beta-\gamma r_{1}\right)<0,
$$

which is a contradiction. Since $u$ and $\varphi_{C_{0}, \tau}$ are ordered at $t_{0}$, comparison implies that they are ordered at any time $t \geqslant t_{0}$.

\section{Outer limit}

We show that the asymptotic behaviour of $u$ near the free boundary is given by a Barenblatt solution with a constant $C_{\star}$ that can be determined in terms of the initial data $u_{0}$.

THEOREM 4.1 Let $N \geqslant 3$ and

$$
C_{\star}=\left(\frac{1}{k(m, N)} \int_{\Omega} H(x) u_{0}(x) \mathrm{d} x\right)^{2(m-1) \beta} .
$$

If $u$ is the weak solution of (1.1), then

$$
\lim _{t \rightarrow \infty} t^{\alpha}\left|u(x, t)-B_{C_{\star}}(x, t)\right|=0
$$

uniformly on sets of the form $\left\{x \in \Omega:|x| \geqslant \delta t^{\beta}\right\}, \delta>0$. 
Proof. We perform the proof in several steps. By a scaling argument, we show that the rescaled solutions converge along subsequences. Then we prove that the limit along any subsequence coincides with $B_{C_{\star}}$. This implies the validity of Theorem 4.1, which is not restricted to any subsequence.

Step 1: Scaling and compactness. We define the family of rescaled solutions

$$
u_{\lambda}(x, t)=\lambda^{\alpha} u\left(\lambda^{\beta} x, \lambda t\right)
$$

where $\alpha$ and $\beta$ are given in 1.2. The Barenblatt functions are invariant under this scaling. Therefore, by the upper bound established in Section 3 the family $\left\{u_{\lambda}\right\}$ is uniformly bounded by some Barenblatt solution for $t \geqslant \bar{t} / \lambda$, a time that tends to zero as $\lambda \rightarrow \infty$. Thus, thanks to the results on compactness for the PME, [8], [26], there is a subsequence $\left\{\lambda_{k}\right\}$ and a function $u_{\infty}$ such that $u_{\lambda_{k}} \rightarrow u_{\infty}$ uniformly on compact subsets of $\mathbb{R}^{N} \backslash\{0\} \times(0, \infty)$. Moreover, $u_{\infty}$ is a local weak solution of the PME in $\mathbb{R}^{N} \backslash\{0\} \times(0, \infty)$.

Step 2: The limit is a Barenblatt solution. We now show that $u_{\infty}$ is a Barenblatt solution. We know from the previous section that there exists a constant $C$ such that

$$
\varphi\left(x, t-t_{0}\right)<u(x, t)<B_{C}(x, t) \quad \text { for } t \text { large. }
$$

If we re-scale this expression and then pass to the limit as $\lambda_{k} \rightarrow \infty$, we find that $u_{\infty}$ is bounded, both from above and below, by Barenblatt solutions,

$$
B_{C_{0}} \leqslant u_{\infty} \leqslant B_{C}, \quad x \neq 0 .
$$

Thus, $u_{\infty}$ is a nontrivial solution of the PME in $\mathbb{R}^{N} \backslash\{0\}, t>0$, which is bounded for all positive times. Hence, since $N \geqslant 3$, the singularity can be removed. Here is a standard proof: take a smooth cutoff function $0 \leqslant \psi \leqslant 1$ that vanishes near $x=0$ and is 1 for $|x| \geqslant 1$ and put $\psi_{r}(x)=$ $\psi(x / r)$. We now write the weak formulation of the PME with respect to a test function $\phi(x, t)=$ $\zeta(x, t) \psi_{r}(x)$ where $\zeta \in C_{c}^{\infty}\left(\mathbb{R}^{N} \times(0, \infty)\right)$. Since $\phi=0$ near $x=0$, this test function is admissible for the solution with a bounded singularity. Since $u_{\infty}$ is bounded for $t \geqslant \tau>0$, the limit $r \rightarrow 0$ shows that it is a solution of the PME for all $t>0$ and $x \in \mathbb{R}^{N}$.

Since $u_{\infty}$ is a local weak solution of the PME bounded for any $t \geqslant \tau>0$, it has an initial trace that is a finite measure [7]. From relation (4.3) we get

$$
\operatorname{supp} B_{C_{0}} \subseteq \operatorname{supp} u_{\infty} \subseteq \operatorname{supp} B_{C},
$$

and hence $\operatorname{supp}\left(u_{\infty}(\cdot, t)\right)$ shrinks to $\{0\}$ as $t \rightarrow 0$. We conclude that the initial trace is a multiple of the delta function. Therefore $u_{\infty}$ is a Barenblatt solution, $B_{C_{\star}}$, with a constant $C_{\star}$ that satisfies $C_{0} \leqslant C_{\star} \leqslant C$.

Step 3: Convergence along subsequences. Using the invariance of Barenblatt functions under this scaling, we have

$$
\left|u_{\lambda_{k}}(y, 1)-B_{C_{\star}}(y, 1)\right|=\lambda_{k}^{\alpha}\left|u\left(\lambda_{k}^{\beta} y, \lambda_{k}\right)-B_{C_{\star}}\left(\lambda_{k}^{\beta} y, \lambda_{k}\right)\right|=\lambda_{k}^{\alpha}\left|u\left(x, \lambda_{k}\right)-B_{C_{\star}}\left(x, \lambda_{k}\right)\right|
$$

for $\lambda_{k}$ large, where $x=\lambda_{k}^{\beta} y$. Thus, the uniform convergence of $u_{\lambda_{k}}$ to $B_{C_{\star}}$ as $\lambda_{k} \rightarrow \infty$ in sets of the form $\{|y| \geqslant \delta\}$ implies, taking $\lambda_{k}=t_{k}$, the result stated in Theorem 4.1 for a subsequence. 
Step 4: Conclusion. The final step consists in showing that, independently of the chosen subsequence, the parameter $C_{\star}$ of the limit function is given by (4.1). Let $H$ be a solution of (1.3). Using the conservation law (2.2) we obtain

$$
\int_{\Omega} H(x) u_{0}(x) \mathrm{d} x=\int_{\Omega} H(x) u\left(x, t_{k}\right) \mathrm{d} x .
$$

In order to estimate the integral on the right hand side we split it into two parts,

$$
\int_{\Omega} H(x) u\left(x, t_{k}\right) \mathrm{d} x=\underbrace{\int_{\left\{|x| \leqslant \delta t_{k}^{\beta}\right\} \cap \Omega} H(x) u\left(x, t_{k}\right) \mathrm{d} x}_{I_{1}}+\underbrace{\int_{\left\{|x| \geqslant \delta t_{k}{ }^{\beta}\right\}} H(x) u\left(x, t_{k}\right) \mathrm{d} x}_{I_{2}}
$$

for any $\delta>0$ and $t_{k}$ large enough. If we bound $u$ by a Barenblatt solution, and use the fact that $H \leqslant 1$, we get

$$
I_{1} \leqslant t_{k}^{-\alpha} \int_{\left\{|x| \leqslant \delta t_{k}^{\beta}\right\} \cap \Omega} F_{C}\left(|x| t_{k}^{-\beta}\right) \mathrm{d} x \leqslant \int_{\{|\xi| \leqslant \delta\}} F_{C}(|\xi|) \mathrm{d} \xi
$$

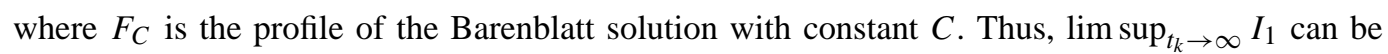
made as small as desired by taking $\delta$ small. In order to compute $I_{2}$ we make again the change of variables $x=\xi t_{k}^{\beta}$ and pass to the limit as $t_{k} \rightarrow \infty$ using the uniform convergence result stated before. Since $\alpha=N \beta$, we obtain

$$
\lim _{t_{k} \rightarrow \infty} I_{2}=\int_{\{|\xi| \geqslant \delta\}} \lim _{t_{k} \rightarrow \infty} H\left(\xi t_{k}^{\beta}\right) u\left(\xi t_{k}^{\beta}, t_{k}\right) t_{k}^{N \beta} \mathrm{d} \xi=\int_{\{|\xi| \geqslant \delta\}} F_{C_{\star}}(|\xi|) \mathrm{d} \xi
$$

Passing to the limit in 4.4], and then letting $\delta \rightarrow 0$, gives

$$
\int_{\Omega} H(x) u_{0}(x) \mathrm{d} x=\int_{\mathbb{R}^{N}} F_{C_{\star}}(|\xi|) \mathrm{d} \xi=M_{C_{\star}}=k(m, N) C_{\star}^{1 / 2(m-1) \beta},
$$

where $k(m, N)$ is given by 3.4. This result does not depend on the particular sequence $\left\{t_{k}\right\}$.

REMARK 1 Theorem 4.1 is also true when we replace $B_{C_{\star}}(x, t)$ by a Barenblatt solution that is centred at $x_{0} \neq 0, B_{C_{\star}}\left(x-x_{0}, t\right)$. This poses an interesting problem, the optimal choice of $x_{0}$.

As a corollary of the outer behaviour we obtain the rate of decay of the mass to its asymptotic limit $M_{C_{\star}}=\int_{\mathbb{R}^{N}} F_{C_{\star}}(|\xi|) \mathrm{d} \xi>0$. The fact that there is a nonzero asymptotic mass is a property that is not true for $N=1,2$.

COROLlary 4.2 Let $N \geqslant 3$ and let $u$ be the weak solution of (1.1). The mass of the solution at time $t, M(t)=\int_{\Omega} u(x, t) \mathrm{d} x$, satisfies

$$
M(t)=M_{C_{\star}}+K t^{-\beta(N-2)}+o\left(t^{-\beta(N-2)}\right),
$$

where

$$
K=C_{\Omega} \int_{\mathbb{R}^{N}} F_{C_{\star}}(|\xi|)|\xi|^{2-N} \mathrm{~d} \xi, \quad C_{\Omega}=\lim _{|x| \rightarrow \infty} \Phi(x)|x|^{N-2},
$$

and $\Phi=1-H$. 
The existence of the limit $C_{\Omega}>0$ is a simple property of harmonic functions on exterior domains that go to zero at infinity; it can be found in [19, Lemma 4.5].

Proof. It is similar to that of Theorem 4.1. Step 4. We deduce from (4.5) that

$$
t^{\beta(N-2)}\left(M(t)-M_{C_{\star}}\right)=t^{\beta(N-2)} \int_{\Omega} u(1-H)=t^{\beta(N-2)} \int_{\Omega} u \Phi .
$$

In order to estimate this last integral we split it into two parts,

$$
\begin{aligned}
& t^{\beta(N-2)} \int_{\Omega} \Phi(x) u(x, t) \mathrm{d} x \\
& =\underbrace{t^{\beta(N-2)} \int_{\left\{|x| \leqslant \delta t^{\beta}\right\} \cap \Omega} \Phi(x) u(x, t) \mathrm{d} x}_{I_{1}}+\underbrace{\left.t^{\beta(N-2)} \int_{\{|x| \geqslant \delta t} \Phi\right\}}_{I_{2}} \Phi(x) u(x, t) \mathrm{d} x
\end{aligned}
$$

for $\delta>0$ and $t$ large enough. Using the estimate $0 \leqslant \Phi(x) \leqslant c_{1}|x|^{2-N}$ and the change of variables $x=\xi t^{\beta}$ we get $0 \leqslant I_{1} \leqslant C \delta^{2}$. For $I_{2}$, we make again the change of variables $x=\xi t^{\beta}$, and get

$$
\begin{aligned}
\lim _{t \rightarrow \infty} I_{2} & =\int_{\{|\xi| \geqslant \delta\}} \lim _{t \rightarrow \infty} \frac{\Phi\left(\xi t^{\beta}\right)|\xi|^{N-2} t^{\beta(N-2)}}{|\xi|^{N-2}} u\left(\xi t^{\beta}, t\right) t^{N \beta} \mathrm{d} \xi \\
& =C_{\Omega} \int_{\{|\xi| \geqslant \delta\}} F_{C_{\star}}(|\xi|)|\xi|^{2-N} \mathrm{~d} \xi .
\end{aligned}
$$

Hence

$$
\begin{gathered}
C_{\Omega} \int_{\{|\xi| \geqslant \delta\}} F_{C_{\star}}(|\xi|)|\xi|^{2-N} \mathrm{~d} \xi \leqslant \liminf _{t \rightarrow \infty} t^{\beta(N-2)}\left(M(t)-M_{C_{\star}}\right) \\
\quad \leqslant \limsup _{t \rightarrow \infty} t^{\beta(N-2)}\left(M(t)-M_{C_{\star}}\right) \leqslant C \delta^{2}+C_{\Omega} \int_{\{|\xi| \geqslant \delta\}} F_{C_{\star}}(|\xi|)|\xi|^{2-N} \mathrm{~d} \xi,
\end{gathered}
$$

from which the result follows by just letting $\delta \rightarrow 0$.

REMARK 2 (Loss of mass) The amount of mass, $M_{L}(u)$, lost in the evolution is given by

$$
M_{L}(u):=\int_{\Omega} u_{0}(x) \mathrm{d} x-\lim _{t \rightarrow \infty} \int_{\Omega} u(x, t) \mathrm{d} x=\int_{\Omega}(1-H(x)) u_{0}(x) \mathrm{d} x>0,
$$

which in terms of $\Phi$ reads

$$
M_{L}(u)=\int_{\Omega} u_{0}(x) \Phi(x) \mathrm{d} x .
$$

Therefore, the influence of the hole structure is felt at the asymptotic level through the projection of the initial data on $\Phi$, which represents in this way the dissipation capacity of $G$. Indeed, this connection is justified by standard potential theory, since $\Phi$ is the harmonic function defined in $\Omega$ that takes value 1 on $\partial \Omega$ and 0 at infinity. In other words, $\Phi$ measures the capacity of $G$ by means of the formula

$$
\operatorname{cap}_{1,2}(G)=\int_{\Omega}|\nabla \Phi|^{2} \mathrm{~d} x .
$$


To end this section, we consider the behaviour of the free boundary of $u$ as $t$ becomes large. For this purpose, we define

$$
m_{+}(t)=\max _{x \in \Gamma(t)}|x|, \quad m_{-}(t)=\min _{x \in \Gamma(t)}|x| .
$$

We also note that the Barenblatt solution $B_{C_{\star}}$ has free boundary $|x|=y_{\star} t^{\beta}$ with

$$
y_{\star}=\left(\frac{2 m C_{\star}}{\beta(m-1)}\right)^{1 / 2} \text {. }
$$

Corollary 4.3 Let $N \geqslant 3$ and let $u$ be the weak solution of (1.1). Then

$$
\lim _{t \rightarrow \infty} \frac{m_{ \pm}(t)}{t^{\beta}}=\left(\frac{2 m C_{\star}}{\beta(m-1)}\right)^{1 / 2}
$$

Proof. (i) The uniform convergence of the rescaled solutions in sets of the form $|x| / t^{\beta} \geqslant \delta>0$, and the shape of the Barenblatt profile $F_{C_{\star}}$, which is uniformly positive in the sets $|x| / t^{\beta} \leqslant y_{\star}-\varepsilon$, imply that given a small $\varepsilon>0$, the expression $t^{\alpha} u(x, t)$ is uniformly positive for all $t$ large enough. This means that

$$
\frac{m_{-}(t)}{t^{\beta}} \geqslant\left(\frac{2 m C_{\star}}{\beta(m-1)}\right)^{1 / 2}-\varepsilon
$$

if $t$ is large enough, $t \geqslant t(\varepsilon)$. In the limit $\varepsilon \rightarrow 0, t \rightarrow \infty$ we get the lower part of estimate 4.9.

(ii) Since the asymptotic mass is $M_{C_{\star}}$ (see Corollary 4.2 , $M\left(t_{1}\right)$ is below $M_{C_{\star}+\varepsilon}$ for some large time $t_{1}$. We consider the solution $\widehat{u}$ of the PME in the whole space $\mathbb{R}^{N}$ with initial data

$$
\widehat{u}\left(x, t_{1}\right)= \begin{cases}u\left(x, t_{1}\right), & x \in \Omega, \\ 0, & x \notin \Omega .\end{cases}
$$

Let $\widehat{\Gamma}(t)$ be the free boundary of $\widehat{u}$ at time $t$ and $\widehat{m}_{+}(t)=\max _{x \in \widehat{\Gamma}(t)}|x|$. Since $\widehat{u}$ is a supersolution of our problem for $t \geqslant t_{1}$, we have $\widehat{m}_{+}(t) \geqslant m_{+}(t)$ for these times. It is well-known [21] that

$$
\lim _{t \rightarrow \infty} \frac{\widehat{m}_{+}(t)}{t^{\beta}}=\left(\frac{2 m}{\beta(m-1)}\left(\frac{M\left(t_{1}\right)}{k(m, N)}\right)^{2(m-1) \beta}\right)^{1 / 2} .
$$

Hence, by the relation 3.3 ,

$$
\lim _{t \rightarrow \infty} \frac{\widehat{m}_{+}(t)}{t^{\beta}} \leqslant\left(\frac{2 m\left(C_{\star}+\varepsilon\right)}{\beta(m-1)}\right)^{1 / 2},
$$

from which the result follows by letting $\varepsilon \rightarrow 0$.

\section{Inner limit. Matching}

We know that $u$ decays as $O\left(t^{-\alpha}\right)$, since $u$ is bounded both from above and from below by functions with such decay. What asymptotic profile do we get in the inner layer if we scale the solution 
according to this size factor? In order to guess an answer we make some formal computations. Let $v=t^{\alpha} u$; then $v$ satisfies the equation

$$
\Delta v^{m}=t^{-2 \beta}\left(t v_{t}-\alpha v\right)
$$

in $\Omega \times(0, \infty)$, with the boundary condition $v=0$ on $\partial \Omega$. Assume for the moment that $t^{-2 \beta+1} v_{t} \rightarrow 0$ as $t \rightarrow \infty$. Then the limit of $v^{m}$ is expected to be a nontrivial solution of the Laplace equation in $\Omega$ with zero Dirichlet boundary data. There is a whole family of solutions to this problem. They are all a constant factor of the solution $H$ of 1.3 . Hence they are determined by their height at infinity. In order to determine this height we use matched asymptotics: the outer limit of the inner expansion should coincide with the inner limit of the outer development.

THEOREM 5.1 Let $N \geqslant 3$ and let $u$ be the weak solution of (1.1). Then the inner asymptotic behaviour of $u$ is given by the stationary state $H_{C_{\star}}$, where

$$
H_{C_{\star}}(x)=C_{\star}^{m /(m-1)} H(x) .
$$

More precisely, given $\varepsilon>0$ there exist $\delta=\delta(\varepsilon)$ and $t_{\text {in }}=t_{\text {in }}(\varepsilon, \delta)$ such that

$$
\left|t^{\alpha m} u^{m}(x, t)-H_{C_{\star}}(x)\right| \leqslant \varepsilon
$$

for all $|x| \leqslant \delta t^{\beta}, x \in \Omega$ and $t>t_{\text {in. }}$.

REMARK 3 In particular, convergence is uniform for $|x| t^{-\beta} \leqslant \lambda(t)$ and $x \in \Omega$ for any positive function $\lambda(t)$ such that $\lim _{t \rightarrow \infty} \lambda(t)=0$.

In order to proceed with the proof, let us first show that there is convergence in time average. In order to simplify the notation we write

$$
W_{T}(\cdot, \tau)=\frac{1}{T} \int_{\tau}^{\tau+T} w(\cdot, s) \mathrm{d} s,
$$

where $w(x, \tau)=t^{\alpha m} u^{m}(x, t)$ with $\tau=\log t$.

Lemma 5.2 Given $\varepsilon>0$ and $T>0$ there exist $\delta=\delta(\varepsilon, T)$ and $\tau_{\text {in }}=\tau_{\text {in }}(\varepsilon, T, \delta)$ such that

$$
\left|W_{T}(x, \tau)-H_{C_{\star}}(x)\right| \leqslant \varepsilon
$$

for all $|x| \leqslant \delta e^{\beta \tau}, x \in \Omega$ and $\tau>\tau_{\text {in }}$.

Proof. Let $\Omega_{\tau}=\Omega \cap \mathcal{B}_{R(\tau)}(0)$ for $R(\tau)=\delta e^{\beta \tau}$, with $\delta>0$ small. Given $\varepsilon>0$ we know from the previous section that there exists a time $\tau_{0}=\tau_{0}(\delta)$ such that

$$
F_{C_{\star}}(\delta)-\varepsilon \leqslant e^{\alpha \tau} u\left(x, e^{\tau}\right) \leqslant F_{C_{\star}}(\delta)+\varepsilon \quad \text { for }|x|=R(\tau), \tau \geqslant \tau_{0},
$$

where $F_{C_{\star}}$ is the profile of the Barenblatt solution, $B_{C_{\star}}$, which gives the outer limit. Then $w$, which is positive and hence classical in $\Omega_{\tau} \times\left(\tau_{0}, \infty\right)$ if $\tau_{0}$ is large enough, satisfies, for $\tau>\tau_{0}$,

$$
\begin{cases}-\Delta w=-e^{-2 \beta \tau}\left(v_{\tau}-\alpha v\right), & x \in \Omega_{\tau}, \\ w=0, & x \in \partial \Omega, \\ w=g, & x \in \partial \mathcal{B}_{R(\tau)}(0) .\end{cases}
$$


The restriction, $g$, of $w$ to $\partial \mathcal{B}_{R(\tau)}(0)$ satisfies

$$
\left(F_{C_{\star}}(\delta)-\varepsilon\right)^{m} \leqslant g(x, \tau) \leqslant\left(F_{C_{\star}}(\delta)+\varepsilon\right)^{m} \quad \text { for } \tau \geqslant \tau_{0} .
$$

Note that $F_{C_{\star}}(\delta)-\varepsilon>0$ if $\delta$ and $\varepsilon$ are small. We want to use the Green's function $\mathcal{G}=\mathcal{G}\left(x_{0}, x ; \Omega_{\tau}\right)$ for the domain $\Omega_{\tau}$, regarding $\tau$ as a frozen coefficient. Namely, given $x_{0} \in \Omega_{\tau}$, we write

$$
-\Delta \mathcal{G}=\delta_{x_{0}} \quad \text { in } \Omega_{\tau}, \quad \mathcal{G}=0 \quad \text { on } \partial \Omega_{\tau},
$$

where $\delta_{x_{0}}$ denotes the Dirac measure at $x_{0}$. Note that the domains we are dealing with, $\Omega_{\tau}$, are bounded and with smooth boundary, hence the Green's function exists. Observe also that $\mathcal{G}$ is differentiable up to the boundary and $\partial \mathcal{G} / \partial v \leqslant 0$ since $\mathcal{G} \geqslant 0$ and $\mathcal{G}=0$ on the boundary.

Then $w$ can be represented as

$$
w(x, \tau)=-\int_{\partial \mathcal{B}_{R(\tau)}(0)} g \frac{\partial \mathcal{G}}{\partial v} \mathrm{~d} \sigma-\int_{\Omega_{\tau}} e^{-2 \beta \tau}\left(v_{\tau}-\alpha v\right) \mathcal{G} \mathrm{d} x .
$$

We claim that, for $T>0$ fixed and $|x| \leqslant R(\tau)$,

$$
\left|\frac{1}{T} \int_{\tau}^{\tau+T} w(x, s) \mathrm{d} s-H_{C_{\star}}(x)\right| \leqslant \varepsilon+c(\varepsilon+\delta)+c \delta^{2}\left(\frac{T+1}{T}\right) .
$$

Indeed, we have

$$
\begin{aligned}
\left|\frac{1}{T} \int_{\tau}^{\tau+T} w(x, s) \mathrm{d} s-H_{C_{\star}}(x)\right| \leqslant & \frac{1}{T}\left(|\underbrace{\mid-\int_{\tau}^{\tau+T} \int_{\partial B_{R(s)}(0)} g \frac{\partial \mathcal{G}}{\partial \nu} \mathrm{d} \sigma \mathrm{d} s-H_{C_{\star}}(x)}_{\left|I_{1}\right|}|\right. \\
& \left.+|\underbrace{\int_{\tau}^{\tau+T} \int_{\Omega_{s}} e^{-2 \beta s}\left(v_{s}-\alpha v\right) \mathcal{G} \mathrm{d} x \mathrm{~d} s}_{\left|I_{2}\right|}|\right) .
\end{aligned}
$$

We start by getting a bound for $I_{1}$ in terms of $T, \delta$ and $\varepsilon$. Since $\partial \mathcal{G} / \partial v$ is nonpositive on $\partial \mathcal{B}_{R(\tau)}(0)$,

$$
\begin{aligned}
I_{1} \leqslant & -\int_{\tau}^{\tau+T} \int_{\partial \mathcal{B}_{R(s)}(0)}\left(F_{C_{\star}}(\delta)+\varepsilon\right)^{m} \frac{\partial \mathcal{G}}{\partial \nu} \mathrm{d} \sigma \mathrm{d} s-H_{C_{\star}}(x) \\
\leqslant & -\int_{\tau}^{\tau+T} \int_{\partial \mathcal{B}_{R(s)}(0)}\left(F_{C_{\star}}^{m}(0)+c_{1} \delta+c_{2} \varepsilon\right) \frac{\partial \mathcal{G}}{\partial \nu} \mathrm{d} \sigma \mathrm{d} s-H_{C_{\star}}(x) \\
= & -\int_{\tau}^{\tau+T} \int_{\partial \mathcal{B}_{R(s)}(0)} F_{C_{\star}}^{m}(0) \frac{\partial \mathcal{G}}{\partial \nu} \mathrm{d} \sigma \mathrm{d} s-H_{C_{\star}}(x) \\
& -(c \varepsilon+c \delta) \int_{\tau}^{\tau+T} \int_{\partial \mathcal{B}_{R(s)}(0)} \frac{\partial \mathcal{G}}{\partial \nu} \mathrm{d} \sigma \mathrm{d} s \\
\leqslant & \int_{\tau}^{\tau+T}\left(\widehat{H}(x ; \tau)-H_{C_{\star}}(x)\right) \mathrm{d} s+c(\varepsilon+\delta) \int_{\tau}^{\tau+T} \int_{\partial \mathcal{B}_{R(s)}(0)}\left(-\frac{\partial \mathcal{G}}{\partial \nu}\right) \mathrm{d} \sigma \mathrm{d} s,
\end{aligned}
$$


where $\widehat{H}$ satisfies the Laplace equation in $\Omega_{\tau}, \widehat{H}=0$ on the fixed boundary $\partial \Omega$ and $\widehat{H}=F_{C_{\star}}^{m}(0)=$ $C_{\star}^{m /(m-1)}$ on $\partial \mathcal{B}_{R(\tau)}(0)$. In the first step we use the inequality

$$
\left(F_{C_{\star}}(\delta)+\varepsilon\right)^{m} \leqslant F_{C_{\star}}^{m}(0)-c \delta+c \varepsilon,
$$

which is valid for $\varepsilon, \delta$ small by Taylor expansion, since $F_{C_{\star}}(0)>0 ; c$ is a constant close to $\left(F_{C_{\star}}^{m}\right)^{\prime}(0)$.

By definition, $H_{C_{\star}}$ converges to $C_{\star}^{m /(m-1)}$ uniformly as $|x| \rightarrow \infty$. Therefore, given $\varepsilon$, we have $\left|H_{C_{\star}}(x)-C_{\star}^{m /(m-1)}\right| \leqslant \varepsilon$ for $|x|=R(\tau)$ and $\tau$ large enough. Hence, by the maximum principle,

$$
\left|\widehat{H}(x, \tau)-H_{C_{\star}}(x)\right| \leqslant \varepsilon
$$

for $|x| \leqslant R(\tau), x \in \Omega$ and $\tau$ large. Thus, since $\int_{\partial \mathcal{B}_{R(s)}(0)}(-\partial \mathcal{G} / \partial \nu) \mathrm{d} \sigma$ is always 1 , independently of the domain (see [10]), we have

$$
I_{1} \leqslant T \varepsilon+T c(\varepsilon+\delta) .
$$

An analogous computation yields $I_{1} \geqslant-T \varepsilon-T c(\varepsilon+\delta)$. Therefore,

$$
\left|I_{1}\right| \leqslant T \varepsilon+T c(\varepsilon+\delta) .
$$

Finding an estimate for $I_{2}$ is a little more involved. It is based on the well-known semiconvexity property

$$
u_{t} \geqslant-\frac{u}{(m-1) t}
$$

(see for example [20]), which in terms of $v$ reads $v_{\tau} \geqslant-2 \beta v /(m-1)$. Since $v$ is bounded and $\mathcal{G}$ is nonnegative,

$$
\begin{aligned}
\left|I_{2}\right| \leqslant & \left|\int_{\tau}^{\tau+T} \int_{\Omega_{s}} e^{-2 \beta s}\left(v_{s}+\frac{2 \beta v}{m-1}\right) \mathcal{G} \mathrm{d} x \mathrm{~d} s\right| \\
& +\left|\int_{\tau}^{\tau+T} \int_{\Omega_{s}} e^{-2 \beta s}\left(\frac{2 \beta}{m-1}+\alpha\right) v \mathcal{G} \mathrm{d} x \mathrm{~d} s\right| \\
\leqslant & \int_{\tau}^{\tau+T} \int_{\Omega_{s}}\left(\left(e^{-2 \beta s} v\right)_{s}+\frac{2 \beta m}{m-1} v e^{-2 \beta s}\right) \mathcal{G} \mathrm{d} x \mathrm{~d} s+c \int_{\tau}^{\tau+T} \int_{\Omega_{s}} e^{-2 \beta s} \mathcal{G} \mathrm{d} x \mathrm{~d} s .
\end{aligned}
$$
Thus,

Let $\mathcal{G}_{\mathcal{B}_{R(\tau)}}$ be the Green's function for the ball $\mathcal{B}_{R(\tau)}(0)$. Comparison yields $\mathcal{G} \leqslant \mathcal{G}_{\mathcal{B}_{R(\tau)}}$ in $\Omega_{\tau}$.

$$
\begin{aligned}
\left|I_{2}\right| \leqslant \underbrace{\int_{\tau}^{\tau+T} \int_{\Omega_{s}}\left(\left(e^{-2 \beta s} v\right)_{s}+\frac{2 \beta m}{m-1} v e^{-2 \beta s}\right) \mathcal{G}_{\mathcal{B}_{R(s)}} \mathrm{d} x \mathrm{~d} s}_{I_{21}} \\
+\underbrace{c \int_{\tau}^{\tau+T} \int_{\Omega_{s}} e^{-2 \beta s} \mathcal{G}_{\mathcal{B}_{R(s)}} \mathrm{d} x \mathrm{~d} s .}_{I_{22}}
\end{aligned}
$$


Since

$$
\mathcal{G}_{\mathcal{B}_{R(\tau)}}\left(x ; x_{0}\right)=c\left(\left|x-x_{0}\right|^{2-N}-\left(\frac{R(\tau)}{\left|x_{0}\right|}\right)^{N-2}\left|\frac{R^{2}(\tau)}{\left|x_{0}\right|^{2}} x_{0}-x\right|^{2-N}\right) \leqslant c\left|x-x_{0}\right|^{2-N},
$$

we can bound the integrals involving $\mathcal{G}_{\mathcal{B}_{R(\tau)}}$ in terms of the estimate

$$
\int_{\mathcal{B}_{R(\tau)}(0)}\left|x-x_{0}\right|^{2-N} \mathrm{~d} x \leqslant c R^{2}(\tau)=c \delta^{2} e^{2 \beta \tau} .
$$

Thus,

$$
I_{22} \leqslant c \int_{\tau}^{\tau+T} e^{-2 \beta s} \int_{\mathcal{B}_{R(s)}(0)} c\left|x-x_{0}\right|^{2-N} \mathrm{~d} x \mathrm{~d} s \leqslant c \delta^{2} T .
$$

In order to estimate the integral $I_{21}$ we split it in two terms as follows:

$$
I_{21} \leqslant c \int_{\tau}^{\tau+T} \int_{\Omega_{s}}\left(e^{-2 \beta s} v\right)_{s}\left|x-x_{0}\right|^{2-N} \mathrm{~d} x \mathrm{~d} s+c \int_{\tau}^{\tau+T} \int_{\Omega_{s}} e^{-2 \beta s}\left|x-x_{0}\right|^{2-N} \mathrm{~d} x \mathrm{~d} s .
$$

The second integral can be estimated in the same way as $I_{22}$. In order to get a bound for the first one, we change the order of integration to get

$$
c \int_{\tau}^{\tau+T} \int_{\Omega_{s}}\left(e^{-2 \beta s} v\right)_{s}\left|x-x_{0}\right|^{2-N} \mathrm{~d} x \mathrm{~d} s=c \int_{\Omega_{\tau+T}}\left|x-x_{0}\right|^{2-N} \int_{\sigma(x)}^{\tau+T}\left(e^{-2 \beta s} v\right)_{s} \mathrm{~d} s \mathrm{~d} x \leqslant c \delta^{2},
$$

for some function $\sigma$.

Summing up, we have

$$
\left|I_{2}\right| \leqslant c \delta^{2}+c \delta^{2} T=c \delta^{2}(T+1) .
$$

Therefore, if $|x| \leqslant \delta e^{\beta \tau}$ and $\tau$ is large enough, then

$$
\frac{1}{T}\left(\left|I_{1}\right|+\left|I_{2}\right|\right) \leqslant \varepsilon+c(\delta+\varepsilon)+c \delta^{2}\left(\frac{T+1}{T}\right),
$$

which proves the claim (5.4), and hence the lemma.

Proof of Theorem 5.1. This is now a calculus lemma. We argue by contradiction. Assume that there is a sequence of points $\left\{\left(x_{n}, \tau_{n}\right)\right\}$ with $\tau_{n} \rightarrow \infty$ such that $\left|x_{n}\right| e^{-\beta \tau_{n}} \rightarrow 0$ as $n \rightarrow \infty$ and $w\left(x_{n}, \tau_{n}\right) \geqslant H_{C_{\star}}\left(x_{n}\right)+2 \varepsilon$. Since $w_{\tau} \geqslant-C w$ with $C$ a positive constant, integrating this expression between $\tau_{n}$ and $\tau_{n}+h$ we get

$$
w\left(x_{n}, \tau_{n}+h\right) \geqslant w\left(x_{n}, \tau_{n}\right) e^{-C h} \geqslant\left(H_{C_{\star}}\left(x_{n}\right)+2 \varepsilon\right) e^{-C h} .
$$

Hence

$$
\begin{aligned}
W_{T}\left(x_{n}, \tau_{n}\right) & \geqslant \frac{1}{T}\left(H_{C_{\star}}\left(x_{n}\right)+2 \varepsilon\right) \int_{0}^{T} e^{-C h} \mathrm{~d} h=\frac{1}{T}\left(H_{C_{\star}}\left(x_{n}\right)+2 \varepsilon\right) \frac{1-e^{-C T}}{C} \\
& \geqslant\left(H_{C_{\star}}\left(x_{n}\right)+2 \varepsilon\right)(1-c T) \geqslant\left(H_{C_{\star}}\left(x_{n}\right)+2 \varepsilon\right)\left(1-\frac{\varepsilon}{H_{C_{\star}}\left(x_{n}\right)+2 \varepsilon}\right) \\
& =H_{C_{\star}}\left(x_{n}\right)+\varepsilon,
\end{aligned}
$$

if $c T<\min \left\{\varepsilon / 2 C_{\star}^{m /(m-1)}, 1 / 4\right\}$. We arrive at a contradiction with the conclusion of Lemma 5.2 


\section{Global formulation}

Theorems 4.1 and 5.1 allow us to write a unified formulation of the asymptotic behaviour of $u$ in terms of a global approximation, $U_{G}$. This global approximation equals zero at the fixed boundary and has compact support. Its moving interface behaves for $t$ large as the free boundary of $B_{C_{\star}}$, and hence as the moving interface of $u$.

TheOREM 6.1 Let $C_{\star}$ be the constant given in (4.5). Let $u$ be the solution of (1.1), and let $U_{G}(x, t)=\left(B_{C_{\star}}(x, t)-t^{-\alpha} C_{\star}^{1 /(m-1)}\left(1-H^{1 / m}(x)\right)\right)_{+}$. Then

$$
\lim _{t \rightarrow \infty} t^{\alpha}\left|u(x, t)-U_{G}(x, t)\right|=0
$$

uniformly for $x \in \Omega$.

Proof. Since

$$
\begin{aligned}
t^{\alpha}\left|u(x, t)-U_{G}(x, t)\right| & \leqslant\left|t^{\alpha} u(x, t)-C_{\star}^{1 /(m-1)} H^{1 / m}(x)\right|+\left|C_{\star}^{1 /(m-1)}-F_{C_{\star}}\left(x t^{-\beta}\right)\right| \\
& =\left|t^{\alpha} u(x, t)-H_{C_{\star}}^{1 / m}(x)\right|+\left|C_{\star}^{1 /(m-1)}-F_{C_{\star}}\left(x t^{-\beta}\right)\right|,
\end{aligned}
$$

Theorem 5.1 implies that, given $\varepsilon>0$, there exist $\delta=\delta(\varepsilon)>0$ and $t_{\text {in }}$ such that $t^{\alpha} \mid u(x, t)-$ $U_{G}(x, t) \mid \leqslant \varepsilon$ if $|x| \leqslant \delta(\varepsilon) t^{\beta}$ and $t \geqslant t_{\text {in. }}$ On the other hand,

$$
t^{\alpha}\left|u(x, t)-U_{G}(x, t)\right| \leqslant t^{\alpha}\left|u(x, t)-B_{C_{\star}}(x, t)\right|+\left|C_{\star}^{1 /(m-1)}\left(1-H^{1 / m}(x)\right)\right| .
$$

It follows from Theorem 4.1 that for any $\delta>0$, and in particular for $\delta(\varepsilon)$, there exists a value $t_{\text {out }}$ such that $t^{\alpha}\left|u(x, t)-U_{G}(x, t)\right| \leqslant \varepsilon$ if $|x| \geqslant \delta(\varepsilon) t^{\beta}$ and $t \geqslant t_{\text {out }}$.

REMARK 4 There is an overlapping region (see Figure 2),

$$
1 / \delta<|x|<\delta t^{\beta}
$$

where $t^{\alpha}\left|B_{C_{\star}}(x, t)-t^{-\alpha} H_{C_{\star}}^{1 / m}(x)\right| \leqslant \varepsilon$ if $\delta$ is small. Indeed,

$$
t^{\alpha}\left|B_{C_{\star}}(x, t)-t^{-\alpha} H_{C_{\star}}^{1 / m}(x)\right| \leqslant\left|t^{\alpha} B_{C_{\star}}(x, t)-C_{\star}^{1 /(m-1)}\right|+\left|H_{C_{\star}}^{1 / m}(x)-C_{\star}^{1 /(m-1)}\right| .
$$

If $|x| \leqslant \delta t^{\beta}$ with $\delta$ small, then $\left|t^{\alpha} B_{C_{\star}}(x, t)-C_{\star}^{1 /(m-1)}\right| \leqslant \varepsilon / 2$. On the other hand, we have $\left|H_{C_{\star}}^{1 / m}(x)-C_{\star}^{1 /(m-1)}\right|<\varepsilon / 2$ if $|x|>1 / \delta$ and $\delta$ is small.

Analogous computations show that, if $|x|$ belongs to the overlapping region, $\delta$ is small and $t$ is large enough, then the inner and the outer behaviour hold simultaneously in that region.

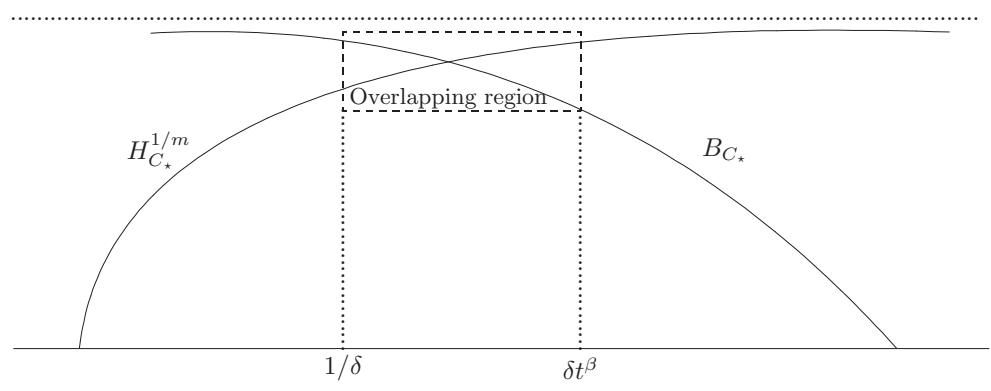

FIG. 2. Overlapping region. 
Convergence to the global approximation also holds in $L^{1}(\Omega)$.

Corollary 6.2 Let $u$ be and $U_{G}$ be as in Theorem 6.1 Then

$$
\lim _{t \rightarrow \infty}\left\|u(x, t)-U_{G}(x, t)\right\|_{L^{1}(\Omega)}=0 .
$$

Proof. There is a constant $C$ such that both $u$ and $U_{G}$ vanish for $|x| \geqslant C t^{\beta}$. Hence, using the previous convergence result, we get

$$
\int_{\Omega}\left|u(x, t)-U_{G}(x, t)\right| \mathrm{d} x=\int_{|x| \leqslant C t^{\beta}}\left|u(x, t)-U_{G}(x, t)\right| \mathrm{d} x \leqslant t^{-\alpha} \varepsilon\left(C t^{\beta}\right)^{N}=C^{N} \varepsilon
$$

for $t$ large enough.

REMARK 5 The $L^{1}$ convergence result can be extended from compactly supported initial data to the whole class of data $u_{0} \in L^{1}(\Omega)$ by a standard density argument (see [21]).

\section{A radial example}

Let the hole $G$ be the ball with radius 1 centred at $0, \mathcal{B}_{1}(0)$, and $u_{0}$ a radial initial data. Since both the domain and the initial data have radial symmetry, the solution $u$ is radial and the original problem (1.1) can be rewritten as

$$
\begin{cases}u_{t}=\left(u^{m}\right)_{r r}+\frac{N-1}{r} u_{r}, & (r, t) \in(1, \infty) \times(0, \infty), \\ u(1, t)=0, & t \in(0, \infty), \\ u(r, 0)=u_{0}(r), & r \in(1, \infty) .\end{cases}
$$

We use a numerical scheme to approximate the solution to this problem. The method has three steps: front tracking, space discretization and time discretization. For the first step we use an algorithm based on ideas from [9], [13]. Then we discretize the $r$ variable with a finite difference scheme and keep $t$ continuous. We solve the resulting ODE with the ODE solver ODE15s provided by MATLAB ${ }^{\circledR}$.

Let us take $u_{0}(r)=((1-r)(r-10))_{+}, N=3$ and $m=2$. Then $\beta=0.2$ and $\alpha=0.6$. We compute the asymptotic constant $C_{\star}$ using formula 4.5 :

$$
\begin{aligned}
C_{\star} & =\left(\frac{1}{k(m, N)} \int_{\Omega} H(x) u_{0}(x) \mathrm{d} x\right)^{2(m-1) \beta} \\
& =\left(\frac{4 \pi}{k(m, N)} \int_{1}^{10}(r-1)^{2}(10-r) r \mathrm{~d} r\right)^{0.4} \approx 9.705,
\end{aligned}
$$

where $H(r)=1-1 / r$ and $k(m, N)=20^{3 / 2} \cdot 8 \cdot \pi / 15 \approx 149.8627$.

First we show, in Figure 3, how the solution evolves with time. The dotted line represents the solution at time $t=10^{4}$, while the dash-dotted line corresponds to $t=10^{5}$. The solid lines represent the solution at intermediate times between $10^{4}$ and $10^{5}$. It can be seen that the solution decreases and that the free boundary increases as time goes by.

Next we run the method until time $t=10^{4}$ and plot the solution in the scales that correspond to the inner and the outer limit. 


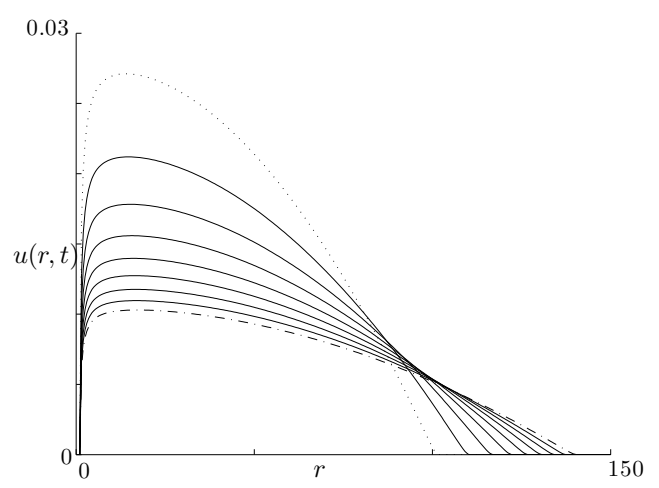

FIG. 3. Evolution of $u$.

In Figure 4 we plot the solution multiplied by $t^{\alpha}$. At first glance, it may seem that there is not convergence to the stationary function $H_{C_{\star}}^{1 / 2}(r)=C_{\star}(1-1 / r)^{1 / 2}$, which is represented by the dotted line. The shape of $t^{\alpha} u$ resembles that of $H_{C_{\star}}$. However, the height is bigger. This is due to the fact that at the time considered the solution has not still lost enough mass.

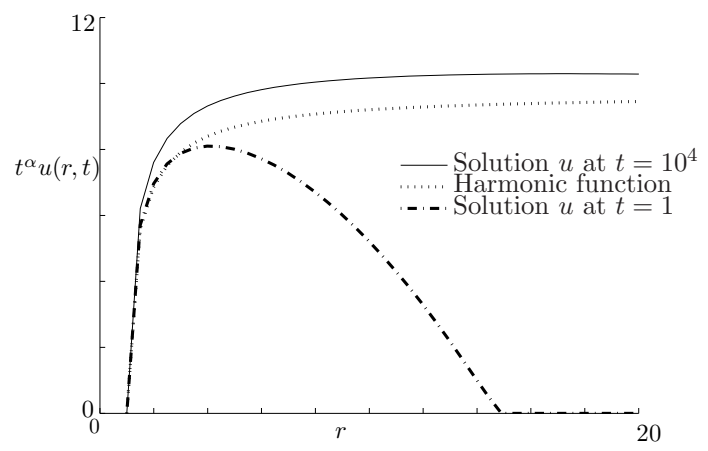

FIG. 4. Inner limit

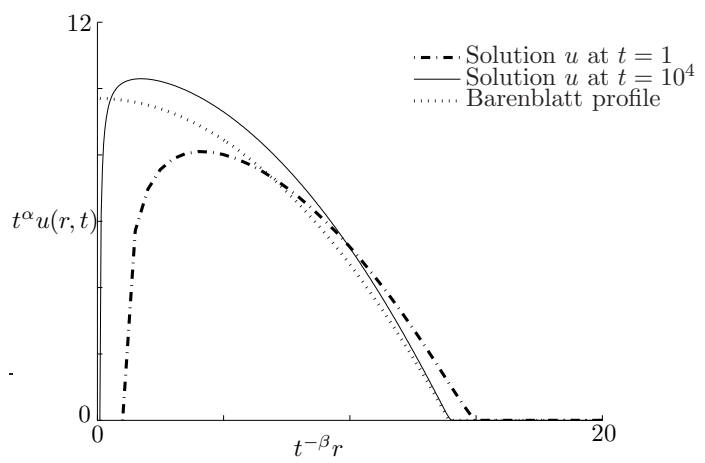

FIG. 5. Outer limit. 


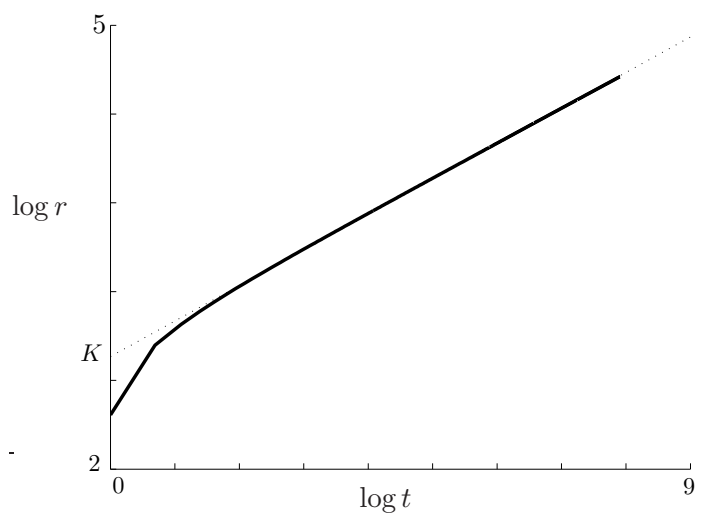

FIG. 6. Free boundary.

Observe that both $H_{C_{\star}}$ and $B_{C_{\star}}$ are known a priori. Hence we do not need to compute them numerically.

Figure 5 shows the outer limit. As in the previous figure, we also notice an accommodation to the shape of the limit profile, in this case the Barenblatt profile that we have denoted by $F_{C_{\star}}$. Notice that at the finite time under consideration the rescaled solution has more mass than the limit. With this scaling, the inner boundary moves to zero. The hole is filled in and it loses its influence on the behaviour of the solution.

From Corollary 4.3 we know that the free boundary grows as $x \sim t^{\beta}$, for $t$ large enough. More precisely, it behaves as $t^{\beta}$ multiplied by an explicit constant. In our case this constant is approximately 13.932. This behaviour can be observed in Figure 6 if we plot the free boundary in logarithmic scale, we see that it converges to a line with slope $\beta=0.2$ and value $K \approx \log (13.932)$ at the origin. In view of Corollary 4.3 , this is the expected result.

\section{Extensions and open problems}

Capacity and removable sets

Given any bounded set $Q$, the capacity of $Q$ is defined as the limit of the capacities of a nested sequence of approximating smooth and bounded domains. An equivalent definition can be given as a variational characterization

$$
\operatorname{cap}_{1,2}(Q)=\inf _{\Phi \in K} \int|\nabla \Phi|^{2}
$$

where $K=\left\{\Phi \in C_{0}^{1}\left(\mathbb{R}^{N}\right): \Phi=1\right.$ on $\left.Q\right\}$ (see for example [10], [17]). We can compute the loss of mass for each of the approximating domains in terms of their capacity. If the limit capacity is zero, then the limit problem does not lose mass. Hence, the solutions of the approximate problems are bounded above by the free solution and the monotone limit has the same mass; therefore, the limit equals the solution of the Cauchy problem defined in the whole of $\mathbb{R}^{N}$. This shows that sets of zero capacity are removable as complements of the domain of the Dirichlet problem. 
Hot spots

A possible extension to this paper would be to determine the hot spots, $\mathcal{H}(t)$, for problem (1.1); i.e. we want to study the movement of

$$
\mathcal{H}(t)=\left\{x \in \bar{\Omega}: u(x, t)=\max _{y \in \bar{\Omega}} u(y, t)\right\}
$$

as $t \rightarrow \infty$. When $N=1$, the behaviour of $\mathcal{H}(t)$ follows directly from the convergence of $u$ to the dipole solution.

Let

$$
\mathcal{H}_{+}(t)=\sup _{x \in \mathcal{H}(t)}|x|, \quad \mathcal{H}_{-}(t)=\inf _{x \in \mathcal{H}(t)}|x| .
$$

If $\xi_{\star}$ is the unique point where the profile of the dipole attains its maximum, then

$$
\lim _{t \rightarrow \infty} \frac{\mathcal{H}_{ \pm}(t)}{t^{1 / 2 m}}=\xi_{\star}
$$

For general $N$ only the case $m=1$ is known. In [14], Ishige proves that if $\Omega=\mathbb{R}^{N} \backslash \mathcal{B}_{R}(0)$ then

$$
\begin{cases}\lim _{t \rightarrow \infty} \frac{\mathcal{H}_{ \pm}(t)}{t^{1 / N}}=\left(2(N-2) R^{N-2}\right)^{1 / N} & \text { if } N \geqslant 3, \\ \lim _{t \rightarrow \infty} \frac{\mathcal{H}_{ \pm}(t)}{(t \log t)^{1 / 2}}=\sqrt{2} & \text { if } N=2 .\end{cases}
$$

In our case, $m>1, N \geqslant 3$, the existence of the global approximation (cf. Theorem 6.1 implies that the hot spots lie in the overlapping region, and we have the bounds

$$
\mathcal{H}_{ \pm}(t) \rightarrow \infty, \quad \mathcal{H}_{ \pm}(t) / t^{\beta} \rightarrow 0
$$

as $t \rightarrow \infty$. The precise behaviour is an open problem. Our conjecture is that they behave like the maximum of the global approximation, i.e., $\mathcal{H}_{ \pm}(t) \sim O\left(t^{2 \beta / N}\right)$. This fits with the linear case mentioned above.

\section{Boundary data with fast decay}

This paper was motivated by the study of the same problem with nonzero boundary conditions on $\partial \Omega$ done by two of the authors in [18]. That paper is mainly concerned with the standard case of data which are positive and constant in time, $u(x, t)=g(x)>0$ for $x \in \partial \Omega, t>0$. However, in Section 11 dealing with conclusions and extensions, we describe how the same methods allow one to treat the case of boundary data which decrease in time in the form

$$
u(x, t) \sim g(x) t^{-\sigma}
$$

and the following result about asymptotic behaviour of the free boundary is stated:

$$
\log (r(t)) \sim \beta_{\sigma} \log (t)
$$

for a $\beta_{\sigma} \geqslant \beta$ that is precisely determined in terms of $m, n$ and $\sigma$ (here, $\beta$ is the exponent of the Barenblatt solution as used above). In particular, for $\sigma<1 / m$ we get $\beta_{\sigma}=m \beta(1-\sigma(m-1))$, 
which is larger than $\beta$, and this exponent is sharp (note that formula (11.4) on page 223 of [18] uses a slightly different notation). It is furthermore stated that for $\sigma \geqslant 1 / m$ the exponent $\beta_{\sigma}$ is exactly $\beta$. We also state that in the limit case $\sigma \rightarrow \infty$ we should recover convergence to the Barenblatt solutions. All these unprecise asymptotic result 11 are easy to prove by putting together the techniques of that paper plus comparison with the behaviour of Barenblatt solutions and suitable self-similar solutions. No further indication is given of asymptotic masses, rates of convergence, inner expansions or capacities. The present paper contributes the details on the precise asymptotics in the case $u=0$ at the boundary ${ }^{2}$

A natural step is now to consider the exact asymptotics when $u=c t^{-\sigma}$ at the boundary, with $\sigma>1 / m$. If $\sigma \geqslant \alpha$, the convergence results presented in this paper still hold, and the free boundary is $O\left(t^{\beta}\right)$. The key point is that we still may use Barenblatt functions as supersolutions. There is still a gap, namely $\sigma \in[1 / m, \alpha)$.

\section{Heat equation and fast diffusion. Other classes of initial data}

Results similar to those contained in this paper (except the ones concerning the free boundary) were obtained by Herraiz [12] for the heat equation $(m=1)$ with initial data $u_{0} \sim C|x|^{-\gamma}, \gamma>N$. Our techniques may be used to extend the results to general data in $L^{1}(\Omega)$. Moreover, they may also be applied to the fast diffusion case $(m<1)$.

The case $m=1, u_{0} \sim C|x|^{-\gamma}, \gamma \leqslant N$ is also considered in [12]. The proofs depend strongly on the linearity of the problem and do not apply to the case $m \neq 1$. On the contrary, our techniques might be used to deal with this problem. The outer behaviour will be given by a selfsimilar solution of the PME in the whole space with the right decay at infinity. These self-similar solutions, constructed in [1], are then matched with a quasi-stationary solution to give the inner behaviour. The hole has no effect on the outer development, since it is negligible, compared with the "big" size of the solution at infinity. Details will be given elsewhere.

\section{Acknowledgements}

All authors were partially supported by Spanish Project MTM2005-08760-C02-01 and ESF Programme "Global and geometric aspects of nonlinear partial differential equations". We thank the referees for suggesting a number of improvements in the presentation.

\section{REFERENCES}

1. Alikakos, N., \& Rostamian, R. On the uniformization of the solutions of the porous medium equation in $\mathbb{R}^{n}$. Israel J. Math. 47 (1984), 270-290. Zbl 0562.35050 MR 0764297

2. Aronson, D. G. The porous medium equation. Nonlinear Diffusion Problems, Lecture Notes in Math. 1224, A. Fasano and M. Primicerio (eds.), Springer, New York (1986), 1-46. Zbl 0626.76097 MR 0877986

3. Aronson, D. G., Crandall, M. G., \& Peletier, L. A. Stabilization of solutions of a degenerate nonlinear diffusion problem. Nonlinear Anal. 6 (1982), 1001-1022. Zbl 0518.35050 MR 0678053

1 This kind of result is an unprecise asymptotic result in the sense that it really does not say that $r(t)=O\left(t^{\beta_{\sigma}}\right)$.

2 The present paper, developing the ideas of [18] was announced there as a paper in preparation, without the name of the first current author. 
4. Aronson, D. G., \& Peletier, L. A. Large time behaviour of solutions of the porous medium equation in bounded domains. J. Differential Equations 39 (1981), 378-412. Zbl 0475.35059 MR 0612594

5. Barenblatt, G., \& ZeL'Dovich, I. B. On dipole-type solution in problems of unsteady filtration of gas in a polytropic system. Prikl. Mat. Mekh. 21 (1957), 718-720 (in Russian).

6. BÉNILAN, P. Opérateurs accrétifs et semi-groupes dans les espaces $L^{p}(1 \leqslant p \leqslant \infty)$. France-Japan Seminar, Tokyo, 1976.

7. Dahlberg, B. E. J., \& Kenig, C. E. Nonnegative solutions of generalized porous medium equations. Rev. Mat. Iberoamericana 2 (1986), 267-305. Zbl 0644.35057 MR 0908054

8. DiBenedetto, E. Continuity of weak solutions to a general porous medium equation. Indiana Univ. Math. J. 32 (1983), 83-118. Zbl 0526.35042 MR 0684758

9. DiBenedetto, E., \& HofF, D. An interface tracking algorithm for the porous medium equation. Trans. Amer. Math. Soc. 284 (1984), 463-500. MR 0743729

10. Gilbarg, D., \& TRUdinger, N. S. Elliptic Partial Differential Equations of Second Order. Grundlehren Math. Wiss. 224, Springer, Berlin (1983). Zbl 0562.35001 MR 0737190

11. Gilding, B. H., \& GonCERZEWICZ, J. Large-time behaviour of solutions of the exterior-domain Cauchy-Dirichlet problem for the porous media euqation with homogeneous boundary data. Monatsh. Math. 150 (2007), 11-39. Zbl pre05133243

12. Herraiz, L. A. A nonlinear parabolic problem in an exterior domain. J. Differential Equations 142 (1998), 371-412. Zbl 0562.35001 MR 0737190

13. Hoff, D. A linearly implicit finite-difference scheme for the one-dimensional porous medium equation. Math. Comp. 45 (1985), 23-33. Zbl 0603.76096 MR 0790642

14. Ishige, K. Movement of hot spots on the exterior domain of a ball under the Dirichlet boundary condition. Preprint.

15. KAMIN, S., \& VÁZqUEZ, J. L. Asymptotic behaviour of solutions of the porous medium equation with changing sign. SIAM J. Math. Anal. 22 (1991), 34-45. Zbl 0755.35011 MR 1080145

16. KING, J. R. Integral results for nonlinear diffusion equations. J. Engrg. Math. 25 (1991), 191-205. Zbl 0753.76168 MR 1096658

17. Lieb, E. H., \& Loss, M. Analysis. Grad. Stud. Math. 14. Amer. Math. Soc. Providence, RI (2001). Zbl 0966.26002 MR 1817225

18. Quirós, F., \& VÁzQuez, J. L. Asymptotic behaviour of the porous media equation in an exterior domain. Ann. Scuola Norm. Sup. Pisa Cl. Sci. (4) 28 (1999), 183-227. Zbl pre01417293 MR 1736227

19. Quirós, F., \& VÁzQuez, J. L. Asymptotic convergence of the Stefan problem to Hele-Shaw. Trans. Amer. Math. Soc. 353 (2001), 609-634. Zbl 0956.35136 MR 1804510

20. VÁzQueZ, J. L. An introduction to the mathematical theory of the porous medium equation. Shape Optimization and Free Boundaries (Montreal, PQ, 1990) NATO Adv. Sci. Inst. Ser. C Math. Phys. Sci. 380, Kluwer, Dordrecht (1992), 347-389. ZZbl 0765.76086 MR 1260981

21. VÁzQUeZ, J. L. Asymptotic behaviour for the porous medium equation posed in the whole space. J. Evol. Equ. 3 (2003), 67-118. Zbl 1036.35108 MR 1977429

22. VÁzQuez, J. L. Dirichlet problem for the porous medium equation in bounded domains. Asymptotic behavior. Monatsh. Math. 142 (2004), 81-111. Zbl 1055.35024 MR 2065023

23. VÁzQUEZ, J. L. Smoothing and Decay Estimates for Nonlinear Diffusion Equations. Equations of Porous Medium Type. Oxford Lecture Ser., Oxford Univ. Press (2006). Z Zbl 0765.76086 |MR 1260981

24. VÁzQuez, J. L. The Porous Medium Equation. Mathematical Theory. Oxford Math. Monogr., Oxford Univ. Press (2007). MR 2286292

25. VÉron, L. Effets régularisants de semi-groupes non linéaires dans des espaces de Banach. Ann. Fac. Sci. Toulouse 1 (1979), 171-200. Zbl 0765.76086 MR 1260981

26. ZIEMER, W. P. Interior and boundary continuity of weak solutions of degenerate parabolic equations. Trans. Amer. Math. Soc. 271 (1982), 733-748. Zbl 0506.35053 MR 0654859 